ERRATUM

THERAPY OF ENDOCRINE DISEASE

\title{
Insulin initiation in patients with type 2 diabetes mellitus: treatment guidelines, clinical evidence and patterns of use of basal vs premixed insulin analogues
}

Allan Vaag ${ }^{1}$ and Sørens Lund ${ }^{1}$

The authors and the journal apologise for an error in the name of the second author of this article published in the February 2012 issue, Vol 166, pp 159-170. The correct name of the author is Søren S Lund.

European Journal of Endocrinology 166565 